\title{
UCRL-TR-155573
}

LAWRENCE

LIVERMORE

NATIONAL LABORATORY

\section{Staged Repository Development Programmes}

T. Isaacs

October 1, 2003 
This document was prepared as an account of work sponsored by an agency of the United States Government. Neither the United States Government nor the University of California nor any of their employees, makes any warranty, express or implied, or assumes any legal liability or responsibility for the accuracy, completeness, or usefulness of any information, apparatus, product, or process disclosed, or represents that its use would not infringe privately owned rights. Reference herein to any specific commercial product, process, or service by trade name, trademark, manufacturer, or otherwise, does not necessarily constitute or imply its endorsement, recommendation, or favoring by the United States Government or the University of California. The views and opinions of authors expressed herein do not necessarily state or reflect those of the United States Government or the University of California, and shall not be used for advertising or product endorsement purposes.

This work was performed under the auspices of the U.S. Department of Energy by University of California, Lawrence Livermore National Laboratory under Contract W-7405-Eng-48. 


\title{
Staged Repository Development Programmes
}

\author{
ITC School of Underground Storage and Disposal
}

\author{
Tom Isaacs \\ Lawrence Livermore National Laboratory \\ isaacs2@llnl.gov
}

October 2003

\section{What is "Staging"}

Programs to manage and ultimately dispose of high-level radioactive wastes are unique from scientific and technological as well as socio-political aspects. From a scientific and technological perspective, high-level radioactive wastes remain potentially hazardous for geological time periods-many millennia-and scientific and technological programs must be put in place that result in a system that provides high confidence that the wastes will be isolated from the accessible environment for these many thousands of years. Of course, "proof" in the classical sense is not possible at the outset, since the performance of the system can only be known with assurance, if ever, after the waste has been emplaced for those geological time periods. Adding to this challenge, many uncertainties exist in both the natural and engineered systems that are intended to isolate the wastes, and some of the uncertainties will remain regardless of the time and expense in attempting to characterize the system and assess its performance.

What was perhaps underappreciated in the early days of waste management and repository program development were the unique and intense reactions that the institutional, political, and public bodies would have to repository program development, particularly in programs attempting to identify and then select sites for characterization, design, licensing, and ultimate development. Reactions in most nations were strong, focused, unrelenting, and often successful in hindering, derailing, and even stopping national repository programs. The reasons for such reactions and the measures to successfully respond to them are still evolving and continue to be the focus of many national program and political leaders.

With such experiences being common, though by no means universal, a number of experienced and thoughtful organizations began to evaluate the causes of program difficulties and to recommend steps that could be taken to take advantage of lessons learned, and to look to principles of operations that seemed to bring better prospects for success. For the last fifteen years or so, beginning with the seminal "Rethinking High-Level Waste" report (1990) produced by an international group of experts drawn together by the U.S. National Academy of Sciences, a rather consistent set of recommendations have been put forward in a number of studies to address these "socio-technical" challenges. These reports (see Bibliography) have as their hallmark the recommendation that, given the unique circumstances summarized above, programs should be developed in a step-wise, phased, or "staged" approach.

The proposition is that an appropriately designed and implemented program will markedly enhance the prospects of program success in meeting both the scientific and technical as well 
as the institutional challenges. It says that "what" we do is important, but "how" we do it is equally important.

As a more recent U.S. National Academy of Sciences report, "One Step at a Time" states: "In summary, Adaptive Staging is a cautious and deliberate decision-making and management process, fully consistent with good engineering practices. It emphasizes continuous learning, both technical and societal, includes scientific and managerial reevaluations and reactions to new knowledge, is responsive to stakeholder input, and is designed to continually improve the project while retaining the option of reversibility as much as possible." (emphasis added; NAS 2003)

\section{Keys Features of Repository Program Staging}

By way of approaching the development of an appropriately staged program, following are some key excerpts from a representative number of reports that provide the basis for the important features and construct.

The 1990 NAS report stated: "There is worldwide scientific consensus that deep geological disposal, the approach being followed in the United States, is the best option for disposing of high-level radioactive waste (HLW). There is no scientific or technical reason to think that a satisfactory geological repository cannot be built. Nevertheless, the U.S. program, as conceived and implemented over the past decade, is unlikely to succeed.

"For reasons rooted in the public's concern over safety and in the implementing and regulatory agencies' need for political credibility, the U.S. waste disposal program is characterized by a high degree of inflexibility with respect to both schedule and technical specifications. The current approach, in which every step is mandated in detail in advance, does have several advantages:

- it facilitates rigorous oversight and technical auditing

- its goals and standards are clear

- it is designed to create a sense of confidence in the planning and operation of the repository; and

- if carried out according to specifications, it is robust in the face of administrative or legal challenge."

"This approach is poorly matched to the technical task at hand. It assumes that the properties and future behavior of a geological repository can be determined and specified with a high degree of certainty. In reality, however, the inherent variability of the geological environment will necessitate frequent changes in the specifications, with resultant delays, frustration, and loss of public confidence...."

"The Board believes, however, that enough has been learned to formulate an approach that can succeed. This alternative approach emphasizes flexibility: time to assess performance and a willingness to respond to problems as they are found, remediation if things do not turn out as planned, and revision of the design and regulations if they are found to impede progress toward the health goal already defined as safe disposal...."

"The neighbors of proposed waste repositories have understandably been alarmed at the prospect of hosting large quantities of a material that needs to be handled with great care. 
Ethical studies in this area underscore two points: (1) the central role of a fair process; and (2) the pervasive problem of promising more certainty than can be delivered."

"The need for a fair process is simply stated: people feel threatened by radioactive waste; and they deserve to be taken seriously in the decision-making process.... The primary goal of the program is to provide safe disposal; a secondary goal is to provide it without any gross unfairness. As a result, the mechanisms of negotiation, persuasion, and compensation are fundamental parts of any program to manage and dispose of radioactive waste-not mere procedural hoops through which program managers must jump." (NAS 1990)

The Academy report went on to then suggest an alternative approach that they believed had attributes better suited to the socio-technical challenges at hand:

"The Board proposes an alternative approach that is built on well-defined goals and objectives, utilizes established scientific principles, and can be achieved in stages with appropriate review by regulatory and oversight bodies and with demonstrated management capabilities. The Board suggests an institutional approach that is more flexible and experimental-in other words, a strategy that acknowledges the following premises:

- Surprises are inevitable in the course of investigating any proposed site, and things are bound to go wrong on a minor scale in the development of a repository.

- If the repository design can be changed in response to new information, minor problems can be fixed without affecting safety, and major problems, if any appear, can be remedied before damage is done to the environment or to public health."

"This flexible approach can be summarized in three principles:

- Start with the simplest description of what is known, so that the largest and most significant uncertainties can be identified early in the program and given priority attention.

- Meet problems as they emerge, instead of trying to anticipate in advance all the complexities of a natural geological environment.

- Define the goal broadly in ultimate performance terms, rather than immediate requirements, so that increased knowledge can be incorporated in the design at a specific site." (NAS 1990)

More recently, a Nuclear Energy Agency (NEA) report on the confidence in the long-term safety of repositories added:

"It is accepted that the novelty and complexity of... tasks mean that detailed planning of the entire repository-development process at the outset of a project is not possible. Although discrete stages can be defined at the outset, detailed planning must proceed iteratively, as information and experience are acquired. In particular, information and experience acquired during the course of one stage can provide a basis for the decision whether to proceed with the next stage, to modify the development programme (perhaps returning to an earlier stage), or in extreme case, to re-assess the programme as a whole."

They added "Measures to enhance confidence" in "The ethical, economical, and political aspects of the appropriateness of the underground disposal option for radioactive waste."

These were: 
- "Careful, incremental approach to decision making, with the possibility of reversing decisions (including, for example, design that facilitates retrievability).

- Wide debate on basic principles (public involvement, collective opinions of the NEA for waste management, IAEA safety fundamentals, etc.).

- Study of the existence and feasibility of alternatives to deep geological disposal." (NEA 1999)

Most recently, the U.S. National Academy of Sciences was asked by the Department of Energy to investigate "The technical, policy, and societal objectives and risks for developing a staged repository system" as well as recommending specific strategies and potential impacts of such an approach.

The Academy report, titled, "One Step at a Time," makes a distinction between linear staging and adaptive staging, making it clear that appropriate staging must be adaptive. The report characterizes linear staging, "...as a management process characterized by a single predetermined path to a selected, completely defined end point, with stages defined principally as milestones where program progress, costs, and schedules are reviewed. The path and end points are reevaluated only if compelling new evidence or other circumstances absolutely require it."

Adaptive Staging, on the other hand, "...begins with a reference framework that can be modified, if necessary, by new information. Decision points mark the transition between stages of project implementation.... Subsequent stages are predicated on the outcomes of previous stages. No single path is therefore recognized from the outset as being fixed; flexibility which allows adaptation of the approach toward agreed overarching goals is maintained throughout." (NAS 2003)

\section{What is "Success"}

Repositories for the disposal of high-level radioactive waste (including spent nuclear fuel) are unique in a number of ways. Some of them present daunting challenges, while others actually allow for meaningful adaptive staging to occur more readily.

There are no licensed geologic repositories for the permanent disposal of high-level radioactive waste. They are first-of-a-kind, and in virtually every country, they are one-of-a-kind. They are complex, relying on a wide mix of natural barriers, engineered barriers, and institutional controls. The programs are long term, taking decades or even centuries from inception through site identification, operation, closure, and post-closure monitoring. They are even longer term in performance, having to isolate wastes from the accessible environment for many millennia, raising concerns about generational equity. There are risks from the handling of the long-life materials, and the public perception and controversy surrounding nuclear waste management often lead to mistrust, lack of agreement on goals, and public and political opposition.

Fortunately, repository programs also offer a number of features that are unique when compared to other nuclear and non-nuclear programs, that provide an opportunity for effective staging.

- Once the waste is emplaced, the system is passive, often requiring no active measures.

- Processes that would lead to wastes returning to the accessible environment are likely to be extremely slow and would not begin for a very long time. 
- The facility only becomes a repository at closure, after a final licensing process. This will occur many decades after initial operation. Until such time, the facility will remain open, the waste can be retrieved, for any reason, and the entire process is reversible.

- There is no arbitrary cutoff for maintaining flexibility. The facility can remain in an open mode, subject to confirmatory testing, surveillance, and retrieval, until future generations are satisfied that they are ready to close the facility.

- Though we will know a great deal at the initial licensing stage, operations and confirmatory testing will greatly add to our knowledge and confidence that the system is performing as expected. The opportunity will exist to optimize the system based upon early experiences, further assure that the system will perform well, design in additional safety measures, or remove the waste.

The measure of success for a repository program might be seen in a more traditional light as getting the most amount of waste into the repository in the least amount of time for the lowest cost, or having all the waste underground in a sealed repository. Rather, the considerations above led the NAS committee to suggest a different definition of a successful repository program, one that reflects the challenges outlined above and may actually result in waste emplacement sooner, with high probability of success, and even with lower costs. The NAS definition is one in which:

- A geologic site and engineered system, judged to be technically suitable using a particular country's accepted regulatory, public, and political processes, have been identified;

- Operational and long-term safety aspects are made consistent with the current scientific understanding of repository systems; safety features are reviewed; and the necessary licenses are granted;

- An ongoing long-term monitoring and observation program designed to substantiate the current scientific understanding of the safety aspects of the repository system is in process;

- Sufficient societal consensus is achieved to allow operations to begin and continue;

- An initial waste emplacement has taken place with plans for reversibility;

- All necessary safety and security measures are set up to emplace additional waste, if decided;

- Procedures and funding arrangements are agreed for either:

- Backfilling (if used), closing, and sealing the repository (if technical and societal confidence in its long-term isolation properties continues); or

- Maintaining capability for long-term control and monitoring, and capability for retrieving wastes, if waste retrieval is necessary for technical or societal reasons.

Given the consequences of such an approach, it is also important to analyze, understand, and accommodate the impacts of adaptive repository staging on the rest of the waste management system, including storage and transportation. There will, of course, also be impacts on costs and emplacement rates, though these will be difficult to quantify, and it may even be difficult to predict whether they will be greater or lesser.

This definition of success can be seen as meeting our obligation to future generations. It is intended to provide them with a clear, safe, and viable option for disposal while those who have benefited from the activities that created the wastes bear the political, institutional, and financial costs. It does not preclude full scale disposal but it does not mandate it either. If everything goes well with such programs, it will be our great-grandchildren, not we, who will decide whether to close such a repository. With such an approach, we leave them a solution and the choice. 


\section{Key Features of an Adaptively Staged Repository Program}

Recognizing the unique features of repository programs and the potential value of adaptively staging, it remains to describe the key features in the management approach. It must be added that, of course, there is no single appropriate process. Rather, program implementers and regulators must develop their programs to reflect the scientific, technical, institutional, political, and cultural aspects within the host nation. Nonetheless, several attributes appear to be common to adaptive staging. Though none is unique to adaptive staging, and indeed they are found in many well run, challenging first-of-a-kind engineering programs, the simultaneous application is what defines Adaptive Staging:

- Commitment to systematic learning - requires a program aimed at the acquisition and incorporation of new scientific, technical, societal, institutional, and operational knowledge during the development process. A central feature is that it intentionally seeks, is open to, and learns from stakeholder input in all knowledge areas. The scientific and management systems must seek out and welcome alternative viewpoints.

- Flexibility - the capability and willingness to reevaluate earlier decisions and redesign or change course if warranted by new information.

- Reversibility - the distinct option to abandon an earlier path and reverse the course of action to a previous stage if new information warrants. Because knowledge will accumulate, the likelihood of reversal is expected to decrease as the program develops.

- Transparency - the decision making process is well documented and available to all stakeholders throughout the process

- Auditability - the complete documentation of the preceding dialogue and the basis for decisions.

- Integrity - implies honesty. It means saying what you will do and doing what you say you will do.

- Responsiveness - requires the implementer to seek, acknowledge, and act on new information and on input from other stakeholders in a timely fashion.

Underlying all of this must be the overriding priority to safety in all programmatic decisions. This most often is translated into the development, continued updating, and central programmatic focus on the "safety case." Though there is no universally accepted definition of a safety case, the Nuclear Energy Agency report, "Confidence in the Long-Term Safety of Deep Geological Repositories (NEA,1999) provides a relevant description:

"The safety case involves descriptions of the possible geological evolutions of the system. Although not capable of proof in a rigorous sense, these descriptions can be supported by relevant observations of the behavior of the various components of the system, while relying on an understanding of the geological history. Furthermore, flexibility should be built into the process of repository development, allowing account to be taken of new understanding and technical information, as well as the demands of societal review.

"The safety case that is provided at a particular stage in the planning, construction, operation or closure of a deep geological repository is part of a broader decision basis that guides the repository-development process...." (NEA 1999)

"Important features of the safety case are: (1) it contains an understandable (to non-experts) explanation of how safety is achieved; (2) it describes the assumptions and concepts that underlie the performance assessment; (3) it discusses directly the uncertainties that could result from limitations in the scientific understanding of the processes and events determining safety; 
and (4) it can use other non-quantitative arguments (such as comparisons with independent lines of evidence, such as historical or natural analogues) to support the plausibility of the safety-relevant behavior of the repository system or its individual components. Two primary roles of the safety case are: (1) to guide the work of the implementer while adapting the program at each stage, and (2) to provide the implementer with a vehicle for making the safety arguments understandable by a wide audience." (NAS 2003)

The safety case will properly vary from site to site and from country to country, but in all cases a robust safety case should have many of the same elements. First it must meet all applicable direct and stringent safety goals required by the regulator. It should assure safety with a set of multiple features, employing both natural and engineered barriers to isolate the wastes. These barriers should be redundant and diverse and provide conservative expectations of meeting or exceeding regulatory requirements and performance goals.

The objective of this robust approach is to provide confidence not only that the repository will perform as required, but that performance will degrade modestly with error-that is, the system should still provide protection should some elements not perform as expected. System design should be such that uncertainty in performance increases slowly with time. The features should be transparent and, to the extent possible, demonstrable. The use of natural and man-made analogues should be employed where appropriate. Retrievability, repairability, and surveillance should be inherent in the program deployment. And the closure decision and subsequent markers should be an explicit decision point. Finally, subjecting the safety case to periodic external and international review provides an additional degree of confidence and transparency.

\section{The Decision Process}

"A structured decision process is an essential part of Adaptive Staging. The purpose of a Decision Point is to assimilate new information, generate options (both anticipated and unanticipated), and make choices for subsequent actions based on acquired data. At Decision Points, the implementer, following stakeholder consultation, determines whether the program will proceed or reiterate a previous stage. Decision Points should be planned at the end of all stages to assess the lessons learned. They can also be introduced in a stage whenever necessary (i.e., if new information warrant reconsideration of program direction)...."

"A Decision Point is not just a "point" in time but a process involving analysis, review, and evaluations, as well as the consequent decisions for future actions. Thus, at a Decision Point the program implementer initiates a process that:

1. systematically gathers, synthesizes, evaluates, and applies the information acquired to date;

2. develops options for the next stage, including explicit consideration of reverting to an earlier stage;

3. evaluates and updates the assessment of the safety of the repository system, in light of the options;

4. makes the findings publicly transparent and available;

5. engages in dialogue with stakeholders;

6. decides on the next stage based on all of the above; and

7. disseminates decisions and their rationales. 


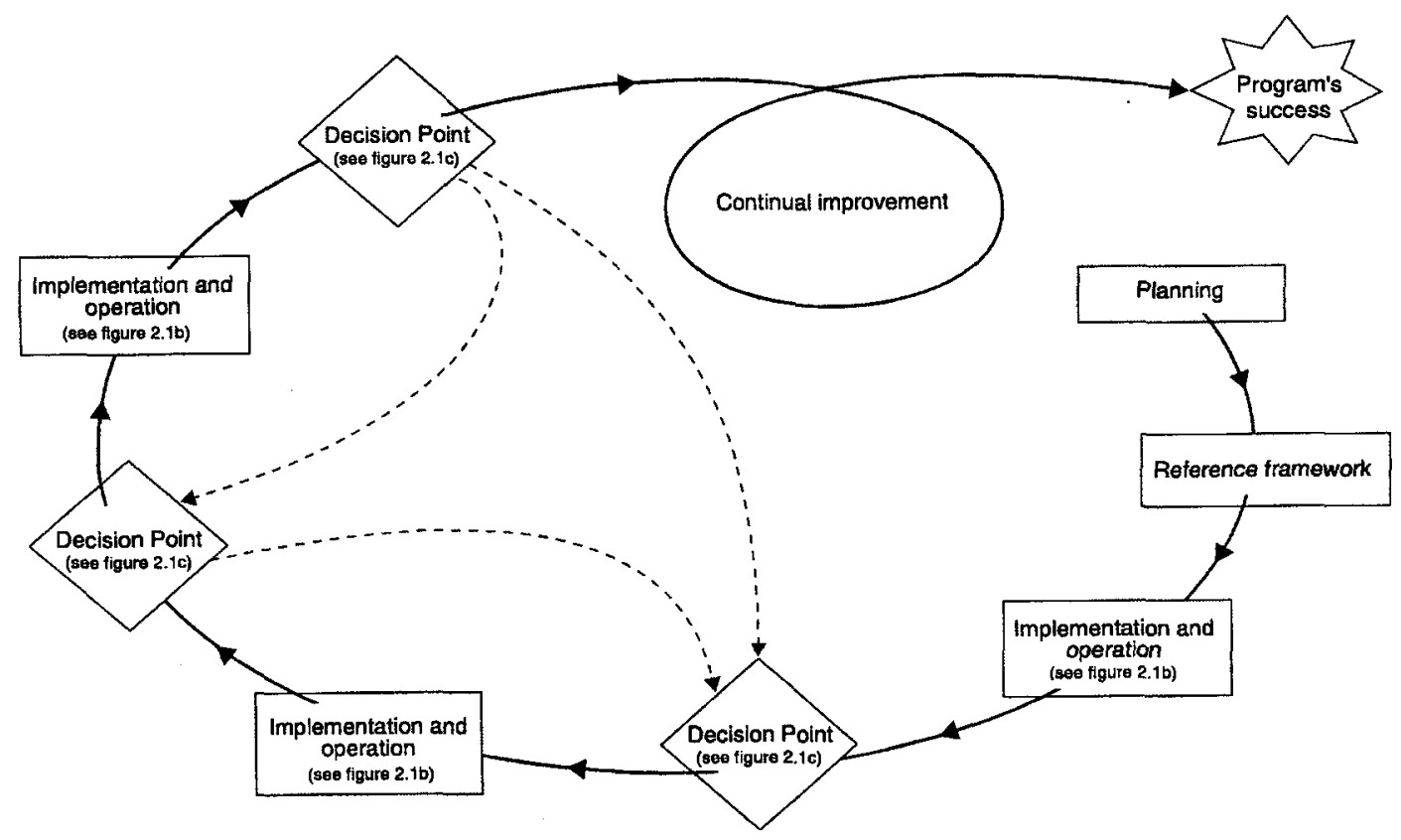

"That is the main reason for introducing these comprehensive formal Decision Points throughout the program, i.e., to ensure that a series of relatively small decisions, each made on narrow grounds, do not lead the program onto an unsound path... In Adaptive Staging, it is not the frequency of Decision Points that matters; it is the readiness to introduce a Decision Point when it is necessary and follow the appropriate decision-making process. The transparency attribute of Adaptive Staging allows all participants to ensure that important decisions are treated as Decision Points. Adaptive Staging does not require program "stops" at each Decision Point. Decision Points can be folded into the schedule so that, when a program is proceeding well, no undue delays are required." (NAS 2003)

\section{Potential Impacts of Staging Repository Development}

There are a number of potential impacts of adopting an Adaptive Staging approach to the repository program. Many of them are intended to have significant benefits to repository performance, costs, schedules, and public acceptance. They also have potential disadvantages that must be carefully considered and accommodated.

Among the most significant affects of Adaptive Staging is the consideration of test facilities, pilot facilities, and demonstration facilities during the early operational phase.

"There are several advantages in implementing test, pilot, and demonstration activities. First, they maximize learning and improve the repository program throughout the years of operation. Second, they may accelerate the schedule and lower costs of first waste emplacement because of the simplified and limited-scale logistical requirements. Third, they lower the early investment in construction because these facilities are less expensive and faster to build than a full-scale repository. Fourth, test, pilot, and demonstration activities address many challenges of geologic repository development; therefore, they improve the chances for program's success. 


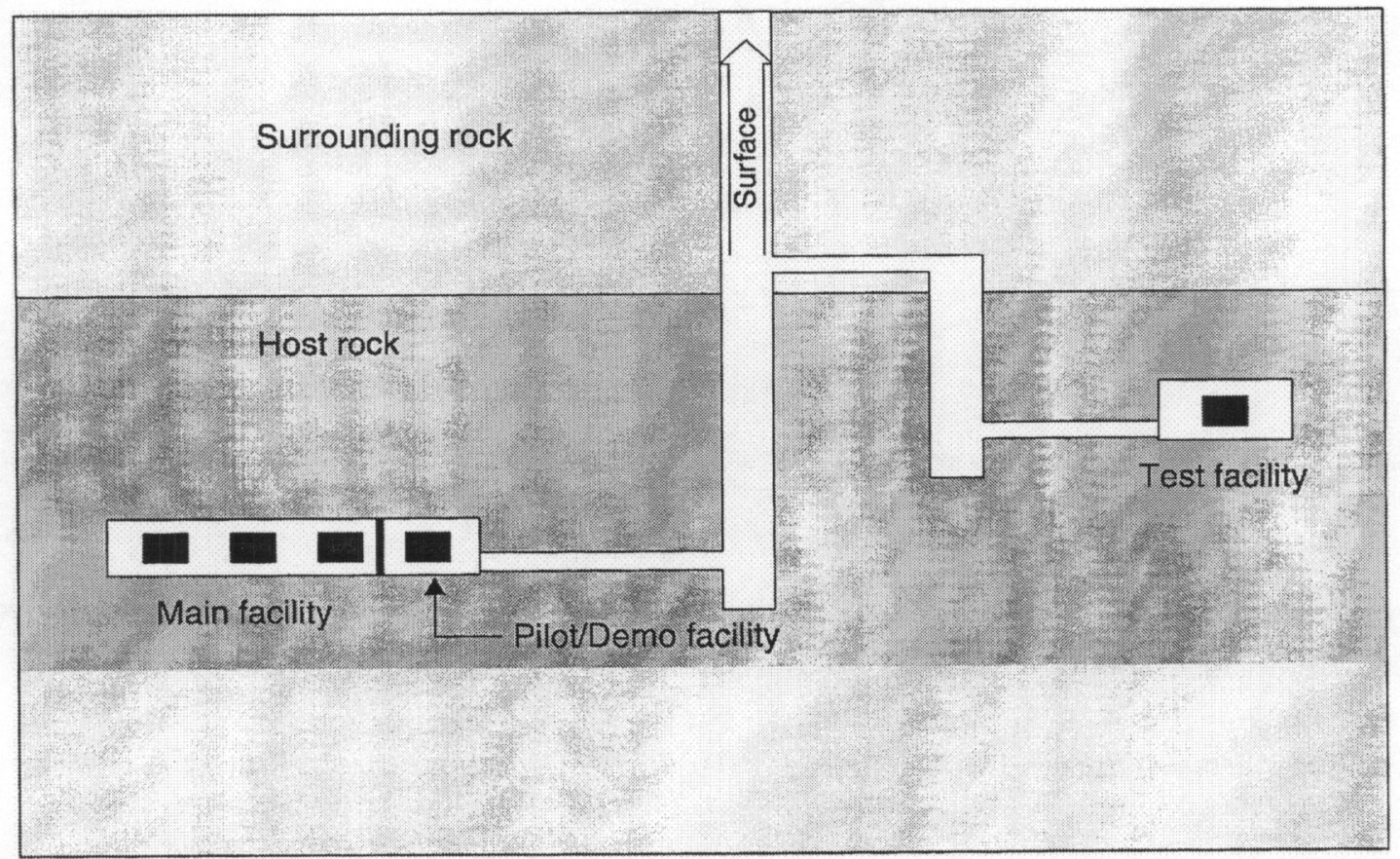

"Disadvantages of implementing test, pilot, and demonstration activities are the additional costs to construct, operate, and maintain the facilities. There will be also additional costs for monitoring the demonstration facility; these costs can be difficult to justify because even intensive short-term monitoring cannot provide direct evidence of long-term safety. Pilot, tests, and demonstration can delay the implementation of full-scale emplacement rates if results call for additional tests or waste retrieval. They can also reduce public confidence in the current understanding of the repository system if inexplicable results are observed-even if these have no obvious safety implications." (NAS 2003)

Other potential impacts include the increase (or decrease) in rate of waste emplacement once the repository is operational. This in turn can have significant impacts on the need for buffer storage requirements, both at waste storage sites (including at reactor storage) as well as at or near the repository itself. It may also impact transportation requirements and ultimately the schedule and process for closing and sealing the repository. All of these changes are likely to have impacts on program schedule and costs that must be carefully considered.

"Adaptive staging may appear to lead to greater costs because of its cautious beginning. However, Adaptive Staging reduces the need to commit prematurely to costly program elements such as materials and technology purchases or full-scale construction. This gradual approach may lead to faster and less expensive resolution of problems. Program revisions made at each stage, based on previous stage experience, may avoid longer-term, irreversible difficulties that could be more expensive to solve and could actually slow progress...." (NAS 2003)

Adaptive Staging will also have impacts on repository closure and post-closure. With Adaptive staging, there will be a flexible schedule for closure and sealing the repository; the repository will (and fortunately can) remain open until residual issues are resolved to the satisfaction of the key stakeholders and there is appropriate agreement that it is safe to close it. This also allows for a post-closure monitoring program to be prescribed and put in place. 


\section{Monitoring and Long-Term Science and Technology Programs}

"A credible, comprehensive monitoring program is an integral part of any management approach for repository development, but takes on increasing value and importance under Adaptive Staging because monitoring allows for systematic learning. The impacts of Adaptive Staging for the monitoring program are:

- monitoring takes on enhanced importance because it is a primary method for obtaining data required to make decisions;

- monitoring activities may increase during the pre-operational and operational phases;

- effort must be devoted to developing a monitoring program that satisfies the attributes of Adaptive Staging;

- mechanisms must be developed to ensure that the monitoring program has sufficient scientific credibility and lack of bias;

- the monitoring program may include a wider array of measurable parameters, including future societal, institutional changes, and economic impacts;

- developing a monitoring program involves costs optimization and organization of financing;

- the monitoring program design must include redundancy; and

- monitoring must link to both program performance confirmation and to the long-term science and technology programs." (NAS 2003)

Likewise, a long-term science and technology program that includes research and development is also an integral part of an adaptively staged geologic program. Key features must include:

- maintaining a continuing long-term science and technology program until closure;

- developing research plans that address the critical technical and societal issues;

- integrating continuously the results;

- retaining qualified staff in the program; and

- integrating the scientists' input into the monitoring program.

"A robust, sustained science and technology program is consistent with the commitment to the systematic learning attribute of Adaptive Staging. The long-term science and technology program reflects the implementer's questioning attitude, searching for vulnerabilities and better approaches to achieving repository goals. In Adaptive Staging, a long-term science and technology program is needed to: (1) reduce known uncertainties; (2) develop the capability for responding to "surprises" (i.e., unknown uncertainties or discoveries); (3) improve or identify weaknesses in the safety case (and help address them) by providing additional evidence that the repository is behaving as predicted; and (4) further develop, refine, or test aspects of the performance assessment methodology." (NAS 2003)

\section{Impacts on Regulation and Licensing}

"Adaptive Staging has the following impacts on the regulatory framework:

- it increases regulatory review steps

- it requires flexibility from the regulator in formulating and applying regulation;

- it requires flexibility in the license amendment process;

- it provides the regulator with increased flexibility to amend regulations if experience warrants; and

- it enhances stakeholder confidence in the regulatory process by increasing transparency." 
"Adaptive Staging may place obstacles in the regulatory process for both the implementer and regulator. From the implementer's point of view, Adaptive Staging may be seen as causing more intensive regulatory oversight which may delay the repository program. From the regulator's point of view, Adaptive Staging requires flexibility and acceptance of the uncertainty involved in permitting or licensing individual stages in a somewhat open-ended program, albeit one supported by a safety case for a full repository." (NAS 2003)

However, Adaptive Staging also has a number of regulatory advantages:

- "It may bring additional information to the regulator about the strengths and weaknesses in the safety case, thereby allowing the regulator to make decisions on the basis of better evidence.

- ...proof that the proposed repository meets any specific set of regulatory standards cannot be demonstrated in the ordinary sense of the word. In Adaptive Staging, this fact is accepted and communicated to regulators, implementers, stakeholders, and the general public. In turn, these parties can help identify additional uncertainties and suggest ways to address them. In this context, Adaptive Staging may provide additional confidence to stakeholders.

- The openness inherent in Adaptive Staging may increase public trust in the regulator and by extension in the repository program. The regulatory body's ability to adopt and utilize a less prescriptive system that involves more judgment is tied to the degree of trust that body enjoys with the broad public. The more trust, the more deference is afforded the regulatory body to exercise judgment instead of relying on prescriptive "yes or no" findings, and the more likely is public acceptance of the regulator's decisions."

Implementers often have a fair amount of discretion in staging program development. However, it is important that early and continuing contacts with the regulator establish a common understanding of the process and the objectives associated with a staged approach.

\section{Public Confidence and Acceptance}

Integral to Adaptive Staging is conducting the repository program in a manner that explicitly works to earn public confidence and achieve public acceptance. Though there can be no guarantees that any approach will be successful, Adaptive Staging has a number of features to improve prospects for success.

"Adaptive Staging can address the issue of public acceptance because of its cautious approach, its structured flexibility, its scheduled periods for reflection and decision-making, and its openness to assimilating all relevant data-including societal and political data. Specifically, Adaptive Staging's attributes of flexibility, transparency, auditability, and responsiveness provide a set of principles and a mechanism for interactive, iterative, stakeholder involvement." (NAS 2003)

"Adaptive Staging encourages and explicitly calls for interaction with stakeholders and the general public at Decision Points. The 1996 National Research Council report, Understanding Risk, concluded that active public participation from the outset and throughout the decisionmaking process is essential to managing risks.... This challenge is summarized in the recommendation to 'get the right participation and get the participation right."'

"Getting the right participation means that the decision-making process should have sufficiently broad participation to ensure that important, decision-relevant information enters the process, 
that all important perspectives are considered, and that the parties' legitimate concerns about inclusiveness and openness are met. Getting the participation right means that the decisionmaking process should satisfy most parties, including stakeholders, that it is responsive to their needs; that their information, viewpoints, and concerns have been adequately presented and taken into account; that parties have been adequately consulted; and that their participation has been able to affect the way risk problems are defined and understood." (NAS 2003)

\section{Summary}

Adaptive Staging suggests an approach to repository program development that reflects the unique challenges associated with the disposal of high-level radioactive waste. The step-wise, incremental, learn-as-you-go approach is intended to maximize the probability of program success, including a redefinition of what success means. The key objective is to come to broad agreement on a program approach that will result in a suitable repository, performing better than required, with the flexibility to adapt to experience and be prepared to change or even reverse direction if conditions merit. The step-wise and transparent approach is intended to foster implementer and regulator behavior that earns the trust and confidence of the many diverse stakeholders and the public by proceeding in a manner that makes it clear that the program is striving to meet their needs and fully address their concerns.

\section{Bibliography}

"One Step at a Time: The Staged Development of Geologic Repositories for High-Level Radioactive Waste," Committee on Principles and Operational Strategies for Staged Repository Systems, National Research Council of the National Academies, The National Academies Press, Washington, D.C., 2003.

"Site Selection Procedure for Repository Sites," Recommendations of the AkEnd Committee on a Site Selection Procedure for Repository Sites, W\&S Druck GmbH, Koln, 2002.

Lidskog, Rolf, and Andersson, Ann-Catrin, "The Management of Radioactive Waste: A Description of Ten Countries, EDRAM (International Association for the Environmentally Safe Disposal of Radioactive Waste, Obrero University Center Man-technology Environment, Obrero, Sweden, 2002.

"Disposition of High-Level Waste and Spent Nuclear Fuel: The Continuing Societal and Technical Challenges," Committee on Disposition of High-Level Radioactive Waste Through Geological Isolation, Board on Radioactive Waste Management, National Research Council of the National Academies, National Academy Press, Washington, D.C., 2001.

"Disposal Concepts for Radioactive Waste," Expert Group on Disposal Concepts for Radioactive Waste, Federal Office of Energy, Bern, Switzerland, 2000.

"Confidence in the Long-Term Safety of Deep Geological Repositories: Its Development and Communication," The OECD Nuclear Energy Agency, Paris, France, 1999.

"Understanding Risk: Informing Decisions in a Democratic Society," National Research Council, National Academy Press, Washington, D.C., 1996. 
Isaacs, Thomas, "What Will Happen to Our Nuclear Waste?" Proceedings of the $2^{\text {nd }}$ MIT International Conference on the Next Generation of Nuclear Power Technology, MIT-ANP-CP-002, Massachusetts Institute of Technology, Cambridge, Massachusetts, 1993.

"Rethinking High-Level Radioactive Waste Disposal," A Position of the Board on Radioactive Waste Management, National Research Council of the National Academies, National Academy Press, Washington, D.C., 1990. 